\title{
Short-term cold acclimation improves insulin sensitivity in patients with type 2 diabetes mellitus
}

Citation for published version (APA):

Hanssen, M. J., Hoeks, J., Brans, B., van der Lans, A. A., Schaart, G., van den Driessche, J. J., Jorgensen, J. A., Boekschoten, M. V., Hesselink, M. K., Havekes, B., Kersten, S., Mottaghy, F. M., van Marken Lichtenbelt, W. D., \& Schrauwen, P. (2015). Short-term cold acclimation improves insulin sensitivity in patients with type 2 diabetes mellitus. Nature Medicine, 21(8), 863-865.

https://doi.org/10.1038/nm.3891

Document status and date:

Published: 01/08/2015

DOI:

10.1038/nm.3891

Document Version:

Publisher's PDF, also known as Version of record

Document license:

Taverne

Please check the document version of this publication:

- A submitted manuscript is the version of the article upon submission and before peer-review. There can be important differences between the submitted version and the official published version of record.

People interested in the research are advised to contact the author for the final version of the publication, or visit the DOI to the publisher's website.

- The final author version and the galley proof are versions of the publication after peer review.

- The final published version features the final layout of the paper including the volume, issue and page numbers.

Link to publication

\footnotetext{
General rights rights.

- You may freely distribute the URL identifying the publication in the public portal. please follow below link for the End User Agreement:

www.umlib.nl/taverne-license

Take down policy

If you believe that this document breaches copyright please contact us at:

repository@maastrichtuniversity.nl

providing details and we will investigate your claim.
}

Copyright and moral rights for the publications made accessible in the public portal are retained by the authors and/or other copyright owners and it is a condition of accessing publications that users recognise and abide by the legal requirements associated with these

- Users may download and print one copy of any publication from the public portal for the purpose of private study or research.

- You may not further distribute the material or use it for any profit-making activity or commercial gain

If the publication is distributed under the terms of Article $25 \mathrm{fa}$ of the Dutch Copyright Act, indicated by the "Taverne" license above, 


\section{Short-term cold acclimation improves insulin sensitivity in patients with type 2 diabetes mellitus}

Mark J W Hanssen ${ }^{1}$, Joris Hoeks ${ }^{1}$, Boudewijn Brans ${ }^{2}$, Anouk A J J van der Lans ${ }^{1}$, Gert Schaart ${ }^{3}$, José J van den Driessche ${ }^{1}$, Johanna A Jörgensen ${ }^{1}$, Mark V Boekschoten ${ }^{4}$, Matthijs K C Hesselink ${ }^{3}$, Bas Havekes $^{5}$, Sander Kersten ${ }^{4}$, Felix M Mottaghy ${ }^{2,6}$, Wouter D van Marken Lichtenbelt ${ }^{1,7}$ \& Patrick Schrauwen ${ }^{1,7}$

\section{Cold exposure may be a potential therapy for diabetes by} increasing brown adipose tissue (BAT) mass and activity. Here we report that $10 \mathrm{~d}$ of cold acclimation $\left(14-15{ }^{\circ} \mathrm{C}\right)$ increased peripheral insulin sensitivity by $\sim 43 \%$ in eight type 2 diabetes subjects. Basal skeletal muscle GLUT4 translocation markedly increased, without effects on insulin signaling or AMP-activated protein kinase (AMPK) activation and only a minor increase in BAT glucose uptake.

Cold-induced adaptive thermogenesis has gained considerable interest due to the 'rediscovery' of BAT in adult humans in 2009 (refs. 1-3). In response to these findings, activation of human BAT has been proposed as a potential treatment for type 2 diabetes (T2D) because such activation leads to the dissipation of energy as heat, which is expected to not only increase energy expenditure but also boost the oxidation of triglycerides and glucose as substrates. Thus it is expected that patients with T2D treated in this way would display not only weight loss but also improvements in lipid and glucose homeostasis ${ }^{4,5}$. Indeed, in rodents enhanced BAT activity has been shown to prevent the development of obesity and diabetes under obesogenic conditions ${ }^{6}$. $\mathrm{We}^{7}$ and others ${ }^{8,9}$ have recently shown that chronic cold exposure (cold acclimation) leads to increases in both BAT quantity and activity in humans, suggesting that BAT can be recruited upon cold acclimation. Here we investigated whether cold acclimation for $10 \mathrm{~d}$ could be used to recruit BAT and improve glucose homeostasis in eight individuals with T2D (Supplementary Table 1). Body weight and clinical blood parameters were unaffected by cold acclimation (Supplementary Table 2), and thyroid-stimulating hormone (TSH) concentrations decreased while free thyroxine (T4) concentrations increased after cold acclimation (Supplementary Table 2).

As a measure of BAT activity, we determined acute cold-induced 2-deoxy-2- $\left[{ }^{18} \mathrm{~F}\right]$ fluoro-D-glucose ( $\left.\left[{ }^{18} \mathrm{~F}\right] \mathrm{FDG}\right)$ uptake by positron emission tomography-computed tomography (PET-CT) scanning (Fig. 1a,b). Upon cold acclimation, $\left[{ }^{18} \mathrm{~F}\right] \mathrm{FDG}$ uptake (standardized uptake values (SUV) $\max$ (data not shown) and $\mathrm{SUV}_{\text {mean }}$ ) in the supraclavicular BAT region increased in all subjects (Fig. 1c). Also, CT radio density (in Hounsfield units) of the BAT depot increased in 7 out of 8 subjects after cold acclimation (Fig. 1d), suggesting that triglyceride content was reduced, indicative of BAT activation ${ }^{10}$. However, we noted that even after cold acclimation the observed $\left[{ }^{18} \mathrm{~F}\right] \mathrm{FDG}$ uptake values in BAT in the T2D patients in our study were very low compared with those in the literature, which were for young, healthy subjects ${ }^{7,11}$. We also measured cold-induced $\left[{ }^{18} \mathrm{~F}\right] \mathrm{FDG}$ uptake in several muscle groups from the cold-acclimated patients. Skeletal muscle $\left[{ }^{18} \mathrm{~F}\right] \mathrm{FDG}$ uptake tended to increase in most of the investigated muscle groups, and the resulting average skeletal muscle $\left[{ }^{18} \mathrm{~F}\right] \mathrm{FDG}$ uptake was indeed increased after cold acclimation (Fig. 1e,f).

We next examined whether glucose uptake in other adipose tissue depots increased upon cold acclimation, which could indicate 'beiging' (conversion of white adipose tissue (WAT) into adipose tissue containing UCP1-expressing adipocytes). However, $\left[{ }^{18} \mathrm{~F}\right] \mathrm{FDG}$ uptake was unaffected by cold acclimation in WAT depots, such as subcutaneous and visceral fat (Fig. 1e). We also performed microarray analysis in subcutaneous WAT biopsies from the human subjects taken before and after $10 \mathrm{~d}$ of cold acclimation and compared it to WAT obtained from cold-acclimated mice. No signs of beiging could be detected in the human WAT samples, and most genes identified as beiging markers were unaffected by the cold acclimation (Fig. 1g). Together these results indicate that after cold acclimation glucose uptake specifically increases in the supraclavicular BAT depot upon acute cold exposure, but BAT activity remains very low in these individuals.

We previously showed that activation of BAT by acute mild cold exposure correlates with cold-induced nonshivering thermogenesis $(\mathrm{NST})^{7,12}$ and that NST increases upon cold acclimation in lean, healthy subjects ${ }^{7}$. Here we found that after, but not before, cold acclimation, acute mild cold exposure increased energy expenditure compared to energy expenditure measured in the thermoneutral zone (TNZ) (Fig. 1h), indicating increased capacity for NST upon cold acclimation. To investigate adaptation to the cold, we determined subjective ratings for sensation, thermal comfort and shivering. All ratings were improved at day 10 of cold acclimation compared to day 3, which was the first day with 6 h of cold exposure (Supplementary Fig. 1). Subjective ratings of hunger, fullness and satiety before and after lunch did not differ between day 10 and day 3 (data not shown). Mean skin and core temperatures, skin blood flow and blood pressure during cold exposure were unaffected by cold acclimation (Supplementary Table 3).

To investigate whether cold acclimation improved glucose homeostasis in individuals with T2D, we used hyperinsulinemic-euglycemic clamps, the gold-standard technique, to determine insulin sensitivity before and after cold acclimation. Of note, clamps were performed

\footnotetext{
${ }^{1}$ Department of Human Biology, School of Nutrition and Translational Research in Metabolism, Maastricht University Medical Center, Maastricht, the Netherlands. ${ }^{2}$ Department of Nuclear Medicine, Maastricht University Medical Center, Maastricht, the Netherlands. ${ }^{3}$ Department of Human Movement Sciences, School of Nutrition and Translational Research in Metabolism, Maastricht University Medical Center, Maastricht, the Netherlands. ${ }^{4}$ Nutrition, Metabolism and Genomics group, Division of Human Nutrition, Wageningen University, Wageningen, the Netherlands. ${ }^{5}$ Department of Internal Medicine, Division of Endocrinology and Metabolism, School of Nutrition and Translational Research in Metabolism, Maastricht University Medical Center, Maastricht, the Netherlands. ${ }^{6}$ Department of Nuclear Medicine, University Hospital Rheinisch-Westfälische Technische Hochschule (RWTH) Aachen, Aachen, Germany. ${ }^{7}$ These authors contributed equally to the study. Correspondence should be addressed to P.S. (p.schrauwen@maastrichtuniversity.nl).
} 
Figure 1 Glucose uptake in the supraclavicular BAT region before and after cold acclimation and WAT beiging. (a) [18F]FDG-PET images after acute cold exposure in one representative individual of the eight in the study before (left) and after (right) cold acclimation. Black arrows indicate supraclavicular BAT activity. (b) Transversal CT, PET and PET-CT fusion slices of the supraclavicular region showing [18F]FDG uptake in BAT locations (white arrows) in the same individual as in a. (c,d) Cold-induced glucose uptake (expressed as SUV mean) (c) and CT radio density (in Hounsfield units) (d) in the supraclavicular BAT region in all individual subjects. (e) Cold-induced glucose uptake in the supraclavicular BAT region, subcutaneous (WAT subc $_{\text {) }}$ ) and visceral WAT $\left(\right.$ WAT $_{\text {visc }}$ ), liver, brain and upper-body skeletal muscle (SM). $n=8,{ }^{*} P<0.05$; error bars, mean \pm s.e.m. (f) Cold-induced glucose uptake in individual skeletal muscle groups used to calculate average SM uptake in e. Er. spinae, erector spinae; Lev. scapulae, levator scapulae. $n=8$; error bars, mean \pm s.e.m. (g) Gene expression changes of selected genes involved in thermogenesis and/or beiging, or suggested as marker genes for beige adipose tissue, in subcutaneous WAT of human subjects with T2D (right column) and subcutaneous WAT from mice subjected to either $10 \mathrm{~d}$ (left column; GSE51080) or $7 \mathrm{~d}$ (middle column; GSE13432) of cold exposure $\left(\sim 5^{\circ} \mathrm{C}\right)$. $P$ values represent intensity-based moderated $t$-statistic (IBMT)-regularized paired $t$-test raw $P$ values. (h) Energy expenditure in thermoneutral conditions and upon acute cold exposure. TNZ, thermoneutral zone. $n=8$; comparisons between data before and after cold acclimation or between thermoneutral and mild cold conditions were analyzed using Wilcoxon signed-rank tests; ${ }^{*} P<0.05$; error bars, mean \pm s.e.m.

at thermoneutrality. Insulin sensitivity was markedly increased after cold acclimation as indicated by on average a $43 \%$ increase in the glucose infusion rate during the clamp (Fig. 2a). In fact, the increase in insulin sensitivity even exceeded the improvements in insulin sensitivity that are seen upon long-term exercise training ${ }^{13}$, which is generally considered to be the best strategy to prevent or treat T2D. Improved whole-body insulin sensitivity was mainly accounted for by a marked increase in insulin-stimulated glucose disposal (Fig. 2b), reflecting improvement of peripheral insulin sensitivity. Basal endogenous glucose production (EGP) was not affected by cold acclimation, but insulin-induced suppression of EGP, reflecting hepatic insulin sensitivity, tended to improve upon cold acclimation (Fig. 2c and Supplementary Table 4). After cold acclimation nonesterified fatty acid concentrations were lower during the clamp (Fig. 2d), which indicated improved adipose tissue insulin sensitivity ${ }^{14}$. Metabolic flexibility, the ability to shift from predominantly fat oxidation in the fasted state to glucose oxidation upon insulin stimulation, also tended to increase (data not shown), most likely as a reflection of improved insulin-stimulated glucose uptake into skeletal muscle ${ }^{15}$.

Because the increase in insulin sensitivity was much more pronounced than anticipated and the activity of BAT remained very low in the treated individuals, we performed detailed analyses of skeletal muscle biopsies obtained after an overnight fast, both before and after $10 \mathrm{~d}$ of cold acclimation. As mitochondrial function is a main determinant of insulin sensitivity in individuals with $\mathrm{T} 2 \mathrm{D}^{16}$, we first examined whether cold acclimation altered skeletal muscle mitochondrial oxidative capacity and/or leak respiration. However, none of the mitochondrial respiration states were affected by cold acclimation (Supplementary Fig. 2a-e). Oxidative phosphorylation complexes and PGC-1 $\alpha$ protein content, markers of mitochondrial content and biogenesis, respectively, were unaffected by cold acclimation (Supplementary Fig. 2f,g). Skeletal muscle fat content was also unaffected by cold acclimation (Supplementary Fig. 2h). It was recently suggested that sarcolipin may regulate futile $\mathrm{Ca}^{2+}$ cycling in skeletal muscle and contribute to nonshivering thermogenesis ${ }^{17}$. However, the protein contents of sarcolipin and the sarcoendoplasmic reticulum calcium-transporting ATPase (Serca) isoform Serca2 were unchanged upon cold acclimation (Supplementary Fig. 2i,j),
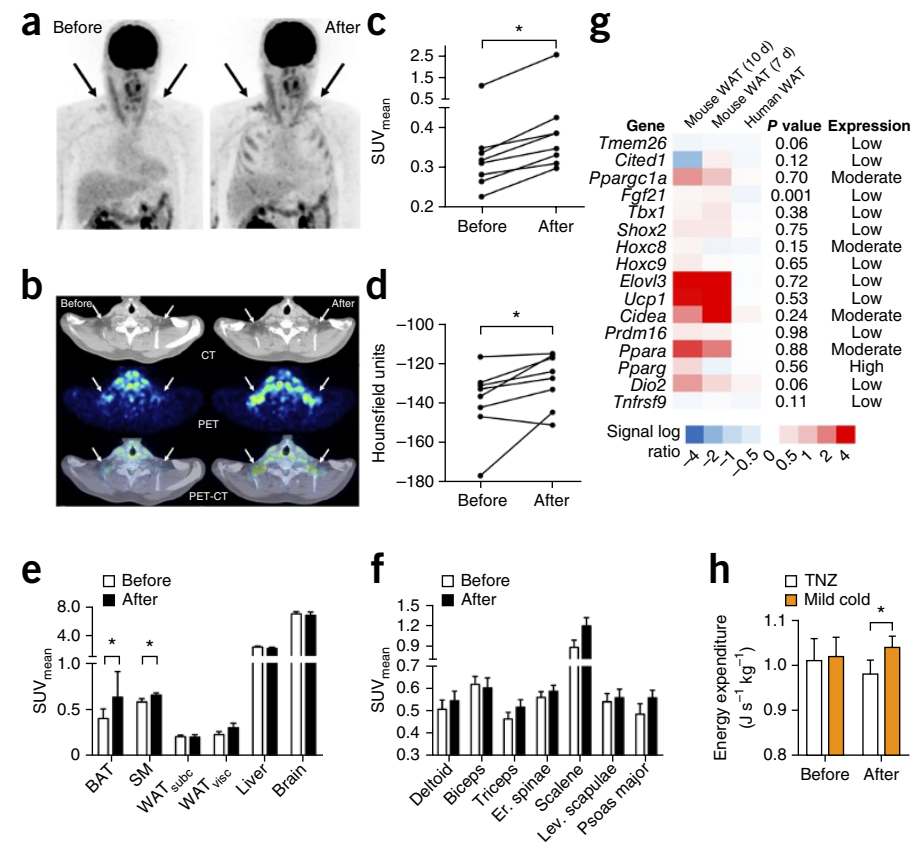

although sarcolipin protein expression correlated with NST after cold acclimation (NST $\left(\mathrm{J} \mathrm{s}^{-1} \mathrm{~kg}^{-1}\right), r=0.893, P=0.007$ ).

Skeletal muscle insulin sensitivity can be more directly affected by enhancement of the insulin-signaling cascade or by activation of $\mathrm{AMPK}^{18}$. We therefore examined biopsies taken before the hyperinsulinemic-euglycemic clamp to determine whether insulin signaling in skeletal muscle was affected by cold acclimation. Notably, phosphorylation of the serine-threonine kinase AKT at both Thr308 (data not shown) and Ser473 (Fig. 2e), as well as total AKT (data not shown), were unaffected by cold acclimation. Furthermore, phosphorylation of the Rab GTPase-activating protein AS160 was also unaffected by cold acclimation (Fig. 2f). We subsequently examined whether altered AMPK activation could contribute to the enhanced insulin sensitivity. However, total AMPK content (data not shown), phosphorylation of AMPK (p-AMPK; from $1,600 \pm 408$ to $1,598 \pm 124 \mathrm{AU}, n=7$, $P>0.05$; data are mean \pm s.e.m.) and p-AMPK/AMPK ratio (Fig. 2g) were unaffected by cold acclimation. In skeletal muscle, GLUT1 and GLUT4 are both involved in glucose uptake. Notably, while GLUT1 protein content was unaffected by cold acclimation (from $0.72 \pm 0.11$ to $0.63 \pm 0.08 \mathrm{AU}, n=7, P>0.05$; data are mean \pm s.e.m.), we observed that total GLUT4 protein content tended to increase after $10 \mathrm{~d}$ of cold acclimation (Fig. 2h). Subsequently, we performed immunostaining of GLUT4 on muscle biopsy sections taken before the clamp to investigate the subcellular distribution of GLUT4 within the muscle cells. Whereas before cold acclimation GLUT4 was evenly distributed over the muscle cell, the pattern was clearly different after cold acclimation, with pronounced staining of GLUT4 at the cell membrane (Fig. 2i) -indicative of translocation of GLUT4 to the membrane to facilitate glucose uptake-occurring even under basal conditions. We quantified GLUT4 localization, which revealed an on average $60 \%$ enrichment of GLUT4 in cell membranes after cold acclimation (Fig. 2j).

The observation of increased GLUT4 in the membrane is consistent with the higher $\left[{ }^{18} \mathrm{~F}\right] \mathrm{FDG}$ uptake in skeletal muscle after cold acclimation (Fig. 1e,f). Therefore, our data suggest that cold acclimation leads to an enrichment of GLUT4 at the sarcolemma, which may facilitate the uptake of glucose. This GLUT4 translocation could not be explained by AMPK activation or improved insulin signaling. Also, subjects did not report 
a

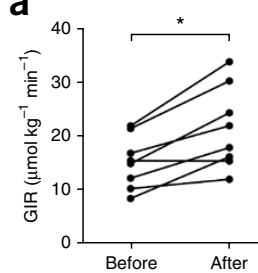

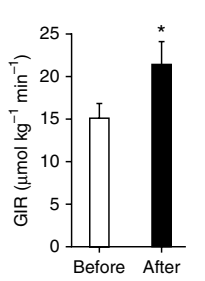

b

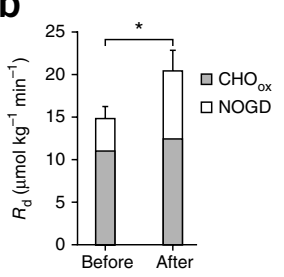

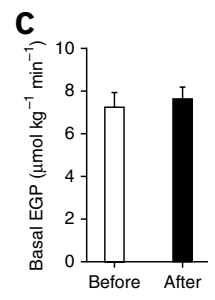
before and after cold acclimation. (a) Individual data (left) and group mean \pm s.e.m. (right) for glucose infusion rate (GIR), corrected for body weight, during hyperinsulinemic-euglycemic clamp; $n=8$. (b) Insulinstimulated glucose disposal $\left(R_{\mathrm{d}}\right)$, as a combination of glucose oxidation $\left(\mathrm{CHO}_{\mathrm{Ox}}\right)$ and nonoxidative glucose disposal (NOGD); $n=8$. (c) Basal (non-insulin-stimulated) endogenous glucose production (EGP; left) and insulin-induced suppression of EGP (\% $\Delta \mathrm{EGP}$ ) (right); $n=8$. (d) Plasma nonesterified fatty acid (NEFA) concentrations during the hyperinsulinemic-euglycemic clamp; $n=8$. (e-h) Western blot data for phosphorylation of AKT at Ser473 (e), phosphorylation of AS160 (f), ratio of phosphorylated AMPK (p-AMPK) and AMPK (g) and GLUT4 $(P=0.063)(\mathbf{h})$, including representative blots of two individuals with T2D, in non-insulin-stimulated skeletal muscle biopsies; $n=7$. (i) Representative images (of 3-5 images per individual) of GLUT4 immunostaining on 5 - $\mu \mathrm{m}$-thick fresh-frozen non-insulin-stimulated skeletal muscle tissue sections from three individuals in the study. Scale bars, $60 \mu \mathrm{m}$. (j) Quantification of average GLUT4 staining intensities at the sarcolemma and the cytosol $(n=7)$. Throughout, comparisons between data before and after cold acclimation were analyzed using Wilcoxon signed-rank tests. ${ }^{*} P<0.05$; error bars, mean \pm s.e.m.

overt shivering in the final days of the cold acclimation and enhanced skeletal muscle GLUT4 translocation after cold acclimation was observed in the overnight-fasted, thermoneutral state. Therefore, alternative mechanisms need to be explored: it has recently been proposed that $\beta$-adrenergic stimulation is able to activate GLUT4 translocation ${ }^{19}$, and daily cold exposure may increase sympathetic activity. Alternatively, it has recently been shown that BAT may release endocrine factors that can engage other metabolic tissues ${ }^{20}$, and it would be worth exploring if some of these endocrine factors have insulin-sensitizing effects on skeletal muscle.

In conclusion, our findings indicate that cold acclimation for $10 \mathrm{~d}$ has very marked positive effects on whole-body and skeletal muscle insulin sensitivity and thereby provide a new avenue to improve the metabolic health of patients with T2D.

\section{METHODS}

Methods and any associated references are available in the online version of the paper.

Accession codes. Gene Expression Omnibus: microarray data have been deposited with accession number GSE67297.

Note: Any Supplementary Information and Source Data files are available in the online version of the paper.

\section{ACKNOWLEDGMENTS}

We thank K. Jardon, E. Broeders, D. Van Moorsel, K. Jansen, M. Visser, R. Hensgens and R. Wierts (Maastricht University Medical Center) for assistance during the experiments and H. Aydeniz, E. Kornips, J. Stegen, W. Sluijsmans, L. Donselaar (Maastricht University Medical Center), W. Wickenhagen (VU University Medical Center Amsterdam) and M. Ackermans (Academic Medical Center Amsterdam) for assistance with the biochemical analyses. The technical support of P. Schoffelen, L. Wouters and M. Souren (Maastricht University Medical Center) is highly appreciated. This work was supported by the EU FP7 project DIABAT (HEALTH-F2-2011-278373 to W.D.v.M.L.) and by the Netherlands Organization for Scientific Research (NWO) (TOP 91209037 to W.D.v.M.L). J.H. is supported by an NWO Vidi grant for innovative research (grant 917.14.358). $\mathbf{f}$
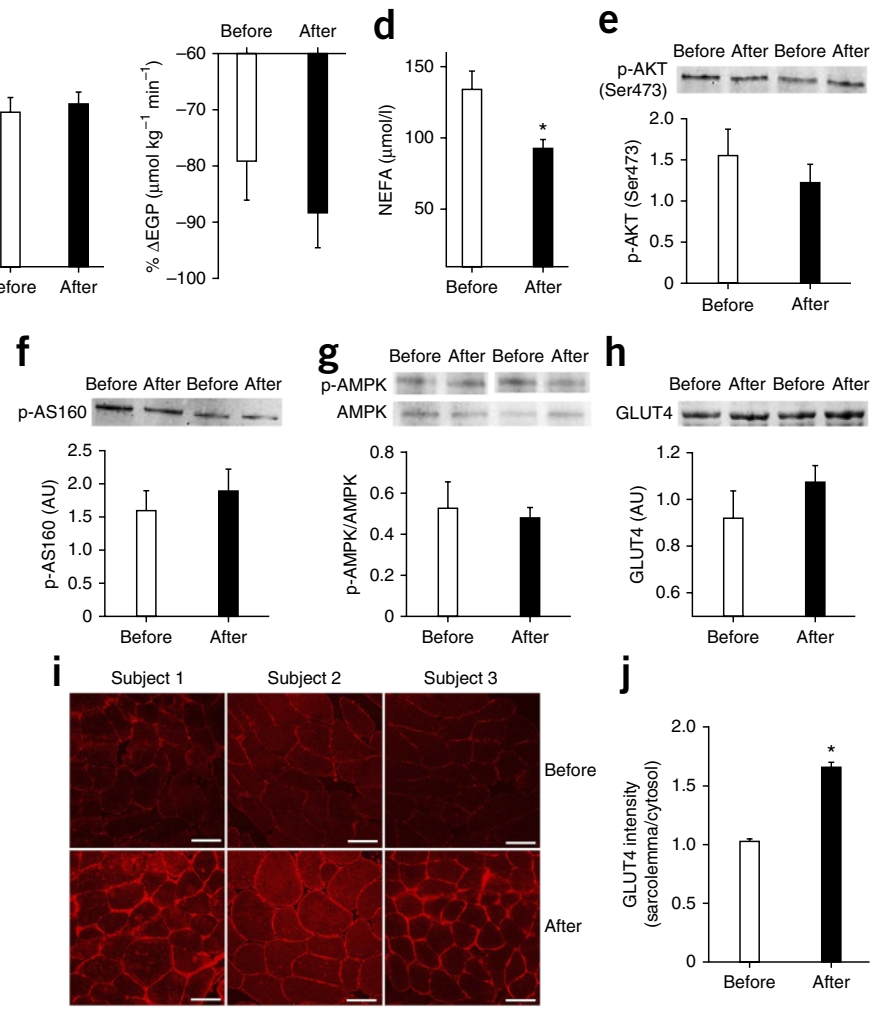

\section{AUTHOR CONTRIBUTIONS}

M.J.W.H. was responsible for study design, data acquisition and data analysis and wrote the manuscript. J.H. contributed to the study design, data acquisition and data analysis. B.B. contributed to data analysis. A.A.J.J.v.d.L. contributed to the study design and data acquisition. J.J.v.d.D., M.V.B., M.K.C.H. and S.K. contributed to data acquisition and data analysis. G.S., J.A.J. and B.H. contributed to data acquisition. F.M.M., W.D.v.M.L. and P.S. contributed to the study design and interpretation of data. All authors contributed to the critical revision of the manuscript and approved the final version.

\section{COMPETING FINANCIAL INTERESTS}

The authors declare no competing financial interests.

Reprints and permissions information is available online at http://www.nature.com/ reprints/index.html.

1. van Marken Lichtenbelt, W.D. et al. N. Engl. J. Med. 360, 1500-1508 (2009).

2. Virtanen, K.A. et al. N. Engl. J. Med. 360, 1518-1525 (2009).

3. Cypess, A.M. et al. N. Engl. J. Med. 360, 1509-1517 (2009).

4. Bartelt, A. et al. Nat. Med. 17, 200-205 (2011).

5. Stanford, K.I. et al. J. Clin. Invest. 123, 215-223 (2013).

6. Townsend, K.L. \& Tseng, Y.H. Trends Endocrinol. Metab. 25, 168-177 (2014).

7. van der Lans, A.A. et al. J. Clin. Invest. 123, 3395-3403 (2013).

8. Yoneshiro, T. et al. J. Clin. Invest. 123, 3404-3408 (2013).

9. Blondin, D.P. et al. J. Clin. Endocrinol. Metab. 99, E438-E446 (2014).

10. Ouellet, V. et al. J. Clin. Invest. 122, 545-552 (2012).

11. Yoneshiro, T. et al. Obesity (Silver Spring) 19, 13-16 (2011).

12. Hanssen, M.J. et al. Diabetologia 58, 586-595 (2015).

13. Meex, R.C. et al. Diabetes 59, 572-579 (2010).

14. Storlien, L., Oakes, N.D. \& Kelley, D.E. Proc. Nutr. Soc. 63, 363-368 (2004).

15. Galgani, J.E. et al. Diabetes 57, 841-845 (2008).

16. Schrauwen-Hinderling, V.B. et al. Diabetologia 50, 113-120 (2007).

17. Bal, N.C. et al. Nat. Med. 18, 1575-1579 (2012).

18. Ruderman, N.B., Carling, D., Prentki, M. \& Cacicedo, J.M. J. Clin. Invest. 123 2764-2772 (2013).

19. Sato, M. et al. Diabetes 63, 4115-4129 (2014).

20. Wang, G.X. et al. Nat. Med. 20, 1436-1443 (2014). 


\section{ONLINE METHODS}

Participants. Eight overweight male individuals with T2D (age $59.3 \pm 5.8$ years, body weight $92.0 \pm 11.7 \mathrm{~kg}$, BMI $29.8 \pm 3.2 \mathrm{~kg} / \mathrm{m}^{2}$, body fat $26.2 \pm 4.0 \%$ ) were included in this study. We performed a power calculation with insulin sensitivity as primary outcome. Based on an expected difference in insulin sensitivity of $20 \%$, with a power of 0.80 and an $\alpha$ of 0.05 (2-sided), we calculated that 11 subjects would be needed ( $G^{*}$ Power 3.1 software, F. Faul, E. Erdfelder, A.G. Lang and A. Buchner, University of Trier, Trier, Germany; http://www.gpower.hhu.de). Since the effects on insulin sensitivity were much more pronounced than anticipated in the power calculation, we terminated the study upon completion of all tests in eight subjects, in agreement with our external monitoring board. All subjects were screened for medical history, and exclusion criteria included uncontrolled hypertension, active cardiovascular disease, liver or kidney dysfunction, smoking and use of insulin, beta-blockers or other medication known to interfere with BAT function. Individuals included in the study were diagnosed with T2D, which was well controlled ( $\mathrm{HbAlc} 50.1 \pm 6.6 \mathrm{mmol} / \mathrm{mol}$ ), at least 1.5 years before the start of the study. All subjects used metformin, and four subjects also used sulfonylurea agents (see Supplementary Table 1 for detailed subject characteristics). Studies were performed between January and September 2014

Study approval. The study was approved by the Ethics Committee of Maastricht University Medical Center and all participants provided written informed consent. Procedures were conducted according to the principles of the Declaration of Helsinki. Trial registration number: NTR4319.

Study design. Body composition was determined by dual X-ray absorptiometry (Discovery A, Hologic, Bedford, MA, USA). Subjects then started the 10-d cold acclimation intervention. Before and after $10 \mathrm{~d}$ of cold acclimation, an individualized cooling protocol was performed, immediately followed by $\left[{ }^{18} \mathrm{~F}\right] \mathrm{FDG}-$ PET-CT scanning (Gemini TF PET-CT, Philips, Eindhoven, the Netherlands) for quantification of BAT activity, as described previously ${ }^{7}$. On a separate day, an abdominal subcutaneous fat biopsy and a muscle biopsy from the vastus lateralis muscle ${ }^{21}$ was taken after an overnight fast. Subsequently, peripheral insulin sensitivity was assessed by a 3 -h hyperinsulinemic-euglycemic clamp, performed at thermoneutrality. During the $10-\mathrm{d}$ cold acclimation protocol, subjects were exposed to an environmental temperature of $14-15^{\circ} \mathrm{C}$ for 10 consecutive days: $2 \mathrm{~h}$ on day $1,4 \mathrm{~h}$ on day 2 , and $6 \mathrm{~h}$ on days 3 through 10 . On day 11 after the start, cold-induced BAT activity measurements were repeated $(\sim 2.5 \mathrm{~h}$ in mild cold). On the following day (day 12), subjects were again exposed to an environmental temperature of $14-15^{\circ} \mathrm{C}$ for $6 \mathrm{~h}$, and this was followed by the second hyperinsulinemic-euglycemic clamp on day 13 . Subjects were instructed to maintain their normal antidiabetic medication use throughout the whole acclimation period, except for the days on which the clamps were performed.

Subjects consumed standardized meals on the evenings before each experimental day and were asked to refrain from heavy exercise at least $48 \mathrm{~h}$ before each of these measurements.

Cold acclimation. During cold acclimation, subjects were dressed in shorts and T-shirts and remained sedentary while staying in the cold room. Food intake in the cold room was kept constant and subjects were instructed not to change their normal dietary regime outside the cold room. Hunger and satiety questionnaires were completed before and after lunch. At selected time points, blood pressure was monitored, VAS scales on sensation, thermal comfort and shivering were completed and incremental AUCs (iAUC) were calculated to determine subjective responses during cold acclimation ${ }^{7}$.

Individualized cooling and PET-CT imaging. Individualized cooling protocols were performed after a $4 \mathrm{~h}$ fasting period. Subjects were wrapped in a waterperfused suit (ThermaWrap Universal 3166, MTRE Advanced Technologies Ltd, Yavne, Israel) and were measured at thermoneutrality for $45 \mathrm{~min}$, after which they were cooled until a temperature just above their shivering point and measured for an additional $30 \mathrm{~min}$ at this temperature. Core and skin temperatures, heart rate, skin perfusion and energy expenditure were measured continuously and blood pressure was measured every $15 \mathrm{~min}$ during the cooling protocol, as described previously ${ }^{7}$. Nonshivering thermogenesis (NST) was calculated as the absolute increase (corrected for body weight) in energy expenditure upon acute mild cold exposure above basal metabolic rate (measured at thermoneutrality). Subsequently, $74 \mathrm{MBq}$ of $\left[{ }^{18} \mathrm{~F}\right] \mathrm{FDG}$ was injected intravenously. One hour after injection the PET-CT scanning protocol started with a low-dose CT scan $(120 \mathrm{kV}, 30 \mathrm{mAs})$. Directly hereafter, a static PET scan (6 to 7 bed positions, 6 min per bed position) was performed ${ }^{22}$. All PET-CT scans were analyzed using PMOD software (version 3.0, PMOD Technologies, Zurich, Switzerland) by both the researcher (M.J.W.H.) and an experienced nuclear medicine physician (B.B.). Fixed volumes of interest (VOIs) were selected in the supraclavicular adipose tissue region (between -10 and -180 Hounsfield units [HU]), subcutaneous and visceral WAT, liver, brain and in the deltoid, biceps, triceps and erector spinae muscles, as described previously ${ }^{23}$. Additionally, $2.67 \mathrm{~cm}^{3}$ VOIs were placed in the scalene, levator scapulae and psoas major muscles, and average uptake in these 7 muscle groups is presented as average skeletal muscle activity. The VOIs were used to compare $\left[{ }^{18} \mathrm{~F}\right] \mathrm{FDG}$ uptake (calculated as $\mathrm{SUV}_{\text {mean }}$ ) and $\mathrm{HU}$ between these tissues and between scans before and after cold acclimation.

Hyperinsulinemic-euglycemic clamp. Hyperinsulinemic-euglycemic $\left(40 \mathrm{mU} \mathrm{m}^{-2} \mathrm{~min}^{-1}\right.$ ) clamps were performed according to DeFronzo ${ }^{24}$, with primed infusion of $\left[6,6{ }^{2} \mathrm{H}_{2}\right]$ glucose $\left(0.04 \mathrm{mg} \mathrm{kg}^{-1} \mathrm{~min}^{-1}\right)$. Steele's single-pool non-steady state equations ${ }^{25}$ were used to calculate rates of glucose appearance $\left(R_{\mathrm{a}}\right)$, glucose disposal $\left(R_{\mathrm{d}}\right)$, nonoxidative glucose disposal (NOGD; mainly reflecting glycogen synthesis) and endogenous glucose production (EGP), as previously described ${ }^{26}$. Basal and insulin-stimulated substrate oxidation was measured by indirect calorimetry (Omnical, IDEE, Maastricht, the Netherlands) and calculated according to Frayn ${ }^{27}$.

Blood analysis. Blood samples were collected before and during the clamp (in the basal and insulin-stimulated states). Plasma metabolites were determined according to standard procedures. Plasma concentrations of glucose (ABX Glucose HK CP Radiometer, Horiba ABX) and nonesterified fatty acids (NEFA-HR set, Wako Chemicals) were determined on a Cobas FARA centrifugal spectrophotometer (Roche Diagnostica). Plasma insulin concentrations were quantified using an immunometric assay (Advia Centaur, Siemens Diagnostics). Serum TSH and T4, and plasma catecholamines, were analyzed as described previously?.

WAT microarray analysis. Abdominal subcutaneous WAT was rinsed from blood, snap frozen in melting isopentane and stored at $-80^{\circ} \mathrm{C}$ until analyzed. Gene expression analysis was performed by microarray and compared to publically available WAT microarray data sets from cold-acclimated mice.

RNA was purified from human fat biopsies using TRIzol (Life Technologies, Calsbad, CA, USA) followed by an additional round of purification with RNeasy Minikit columns (Qiagen, Venlo, the Netherlands). RNA quality was assessed using RNA 6000 nanochips on the Agilent 2100 bioanalyzer (Agilent Technologies, Amsterdam, the Netherlands). Purified RNA (100 ng) was labeled with the Affymetrix WT PLUS reagent kit (Affymetrix, Santa Clara, CA, USA) and hybridized to an Affymetrix Human Gene 1.1 ST array plate (Affymetrix, Santa Clara, CA, USA). Hybridization, washing and scanning were carried out on an Affymetrix GeneTitan platform according to the manufacturer's instructions. Arrays were normalized using the robust multiarray average method ${ }^{28,29}$. Probe sets were defined according to Dai et al. ${ }^{30}$. In this method probes are assigned to Entrez IDs as a unique gene identifier. The $P$ values were calculated using an intensity-based moderated $t$-statistic (IBMT) ${ }^{31}$. The microarray data have been submitted to the Gene Expression Omnibus (accession number GSE67297). Expression changes in the subcutaneous white fat depot of mice subjected to either $10 \mathrm{~d}$ or $7 \mathrm{~d}$ of cold exposure ( $\sim 5$ degrees) were extracted from publicly available microarray data sets (GSE51080 and GSE13432) using the analysis pipeline described above.

Ex vivo skeletal muscle mitochondrial respiration. A portion of the muscle biopsy was directly frozen in melting isopentane and stored at $-80{ }^{\circ} \mathrm{C}$ until assayed. Another portion ( $30 \mathrm{mg}$ ) was immediately placed in ice-cold preservation medium (BIOPS, OROBOROS Instruments, Innsbruck, Austria) and used for the preparation of permeabilized skeletal muscle fibers $(\sim 2.5 \mathrm{mg}$ wet weight $)^{32}$, which were analyzed for mitochondrial oxidative capacity using an 
oxygraph (OROBOROS Instruments) according to Hoeks et al. ${ }^{33}$. In separate experiments, mitochondrial leak respiration was measured as the residual respiration following addition of $1 \mu \mathrm{g} / \mathrm{ml}$ of the ATP synthase-inhibitor oligomycin, using pyruvate $(5 \mathrm{mM})$ as a substrate (in the presence of $4 \mathrm{mM}$ malate). All oxygen consumption measurements were performed in quadruplicate. One muscle biopsy failed after cold acclimation; therefore, values for 7 subjects are presented.

Muscle biopsy analyses. Protein expression was determined by western blotting according to standard procedures. Primary antibodies (AKT, phospho-AKT(Ser473), phospho-AKT (Thr308), phospho-AS160, phosphoAMPK, AMPK(Thr172) and GLUT1; diluted 1:1,000) were all from Cell Signaling Technology, Bioké, Leiden, the Netherlands and were all detected using a horseradish peroxidase-conjugated secondary swine anti-rabbit IgG antibody (DAKO, Glostrup, Denmark; diluted 1:2,000) and measured using enhanced chemiluminescence (Pierce, Thermo Scientific, Rockford, IL, USA).

Primary antibodies directed against GLUT4 (Santa Cruz, BioConnect, Huissen, the Netherlands) UCP3, OXPHOS-cocktail, Serca2 ATPase (all from Abcam, BioConnect); PGC1 $\alpha$ (Calbiochem, Darmstadt, Germany), sarcolipin (Millipore, Schiphol-Rijk, the Netherlands) and $\alpha$-sarcomeric actin (loading control; Sigma, Zwijndrecht, the Netherlands) were detected using appropriate secondary antibodies conjugated with IRDye 680 or IRDye800 and detected with the Odyssey Near Infrared System (Licor, Westburg, Leusden, the Netherlands). Intramyocellular lipid (IMCL) content was determined in fresh cryosections $(5 \mu \mathrm{m})$ by Oil red O staining combined with fibertyping and immunolabeling of the basal membrane marker laminin, as described previously ${ }^{34}$.

For GLUT4 imaging, double immunofluorescence assays were performed on $5-\mu \mathrm{m}$-thick fresh-frozen tissue sections, which were fixated for $15 \mathrm{~min}$ with $3.7 \%$ formaldehyde in PBS and then treated for 5 min with $0.5 \%$ Triton X-100 in PBS. Sections were incubated overnight at $4{ }^{\circ} \mathrm{C}$ with a mix of primary antibodies directed to GLUT4 (Santa Cruz, BioConnect, Huissen, the Netherlands) and laminin (Sigma, Zwijndrecht, the Netherlands). After three washing steps with PBS, Alexa Fluor555- and Alexa Fluor488-conjugated secondary antibodies were incubated for $45 \mathrm{~min}$ at room temperature. After a final washing step with PBS, sections were mounted in Mowiol. Images were observed using a Nikon E800 fluorescence microscope with NIS-elements Imaging Software (Nikon
Europe BV, Amsterdam, the Netherlands) and were captured with identical exposure time and gain settings in 'before' and 'after' conditions. Without any adjustments with respect to color intensity, brightness or contrast, RGB-stacked images were quantified using the Plot Profile tool in ImageJ. Thus, we measured the intensity of GLUT4-dependent signals (16 bits) throughout the sections. Measured data on intensity were used to generate overlying plots of GLUT4 and laminin. The GLUT4-derived staining intensity at the membrane was quantified at multiple locations per individual $(13.7 \pm 2.9$ locations before and $15.4 \pm 3.2$ locations after cold acclimation, respectively) in randomly chosen crosssections of muscle biopsies, and was divided over the mean GLUT4 intensity in 10 pixels located in the cytosol of the very same cell. Thus, a score $>1.0$ implied that relatively more GLUT4 was detected in the membrane than in cytosolic regions and hence reflected GLUT4 translocation.

Statistical analysis. Statistical analyses were performed with PSAW Statistics 20.0 for MAC (SPSS). Nonparametric paired-sample Wilcoxon signed-rank tests were used to compare findings before and after cold acclimation and between thermoneutral and mild cold conditions. Spearman rank correlations were used to identify correlations between variables. $P$ values $<0.05$ were considered statistically significant.

21. Bergström, J., Hultman, E. \& Roch-Norlund, A.E. Scand. J. Clin. Lab. Invest. 29 , 231-236 (1972)

22. Vosselman, M.J. et al. Diabetes 61, 3106-3113 (2012).

23. Vosselman, M.J. et al. Am. J. Clin. Nutr. 98, 57-64 (2013).

24. DeFronzo, R.A., Tobin, J.D. \& Andres, R. Am. J. Physiol. 237, E214-E223 (1979).

25. Steele, R. Ann. NY Acad. Sci. 82, 420-430 (1959).

26. Phielix, E. \& Mensink, M. Physiol. Behav. 94, 252-258 (2008)

27. Frayn, K.N. J. Appl. Physiol. 55, 628-634 (1983).

28. Bolstad, B.M., Irizarry, R.A., Astrand, M. \& Speed, T.P. Bioinformatics 19, 185-193 (2003).

29. Irizarry, R.A. et al. Nucleic Acids Res. 31, e15 (2003).

30. Dai, M. et al. Nucleic Acids Res. 33, e175 (2005).

31. Sartor, M.A. et al. BMC Bioinformatics 7, 538 (2006).

32. Phielix, E. et al. Diabetes 57, 2943-2949 (2008).

33. Hoeks, J. et al. Diabetes 59, 2117-2125 (2010).

34. Koopman, R., Schaart, G. \& Hesselink, M.K. Histochem. Cell Biol. 116, 63-68 (2001) 